\title{
Blood lactate threshold and type II fibre predominance in patients with exertional heatstroke
}

Yaw-Don Hsu, Wei-Hwa Lee, Ming-Key Chang, Shang-Der Shieh, Wen-Long Tsao

\begin{abstract}
Objectives-Severe damage to skeletal muscle is usually seen in patients with exertional heatstroke. Thirty seven young military recruits with exertional heatstroke in Taiwan from 1992 to 1995 were studied to evaluate changes in muscle pathology and blood lactate with exercise. Methods-A biopsy sample of the vastus lateralis was taken from recruits within 10 days of the initial presentation. Results were compared with those from 15 controls matched for age and sex. During the recovery period, $90-150$ days after exertional heatstroke, 29 patients participated in a constant work load test on the treadmill to assess their blood lactate threshold, and a second biopsy sample was taken. Each biopsy was examined histologically for pathology, distribution of fibre types, and fibre diameter.
\end{abstract}

Results-Twenty four of the 37 patients with exertional heatstroke developed rhabdomyolysis and 18 of these had type II fibre predominance in their muscle biopsy. The patients with type II fibre predominance had a higher tendency to develop rhabdomyolysis $\left(\chi^{2}=6.84\right.$, $P<0.01)$. The time required to reach a blood lactate threshold during a constant treadmill work load after recovery was significantly shorter in the patients with exertional heatstroke who had type II fibre predominance $(P<0.01)$. There was a positive correlation between the highest value of blood lactate and the percentage of type II fibres in all tested subjects $(r=$ $0.82, P<0.01$ ).

Conclusion-Patients with type II fibre predominance are more susceptible to exertional heatstroke and tend to have a higher blood lactate concentration and a shorter time to reach blood lactate threshold under a treadmill load test.

(F Neurol Neurosurg Psychiatry 1997;62:182-187)

Keywords: exertional heatstroke; type II fibre predominance; blood lactate threshold

Rhabdomyolysis can be induced by many different factors, ${ }^{1}$ among them, heatstroke and exercise are the most common factors in military recruits. An underlying disorder in skeletal muscle, such as mitochondrial disorder, ${ }^{2}$ glycolytic disorder, ${ }^{34}$ lipid metabolic disorder, ${ }^{56}$ inflammatory myopathy, ${ }^{7}$ and others ${ }^{8-10}$ should also be considered. Great individual variability exists in the ability to sustain heat stress and prolonged exercise. Epstein ${ }^{11}$ mentioned that low tolerance to heat and exercise may result from functional factors, or from individual congenital or acquired myopathy factors. Strydom ${ }^{12}$ thought that a sedentary lifestyle (low physical fitness) was one of the main functional factors in heat intolerance. Besides low physical fitness, the muscle itself is another important factor provoking exertional heatstroke. Previous findings on exercise endurance suggested that a different percentage of fibre types in muscle had a different exercise endurance. ${ }^{13 \cdot 15}$ Glenmark et al ${ }^{16}$ reported that the morphological adaptation of the fibres in humans is related to muscle strength. Therefore, the study of fibre type in patients with exertional heatstroke should help to understand the relation between exercise endurance and the pathogenesis of rhabdomyolysis in exertional heatstroke.

As well as muscle biopsy, measurement of blood lactate threshold is a rapid method of evaluating endurance capacity. In the past, maximal oxygen uptake was considered to be the best way to determine endurance capacity. This method is, however, only valid in assessing short term endurance work loads. A better measure to ascertain medium term and long term endurance has proved to be the determination of the aerobic-anaerobic threshold. ${ }^{17}$ In the present study, we used the blood lactate threshold during exercise to predict the endurance capacity, particularly in the recovery period, and tried to correlate this with different changes in percentages of muscle fibre types.

\section{Subjects and methods}

SUBJECTS

In the past three years, thirty seven military recruits with exertional heat stroke were admitted to Tri-Service General Hospital and had muscle biopsies within 10 days of the episode and another biopsy after recovery. All were men, 19 to 23 (mean 20.4 (SD) $1 \cdot 1$ ) years of age. Their physical condition was reviewed in detail. None had a history of heat exhaustion, heat stroke, childhood febrile seizures, or malignant hyperthermia before this episode. Information on physical activity three months before the recruitment was obtained. They were non-athletic (non-active) students before being recruited into the army. An intensive military training had been sched- 
uled for all of the recruits for at least five days before the heatstroke.

The criteria used to verify exertional heatstroke were similar to those utilised in other studies $^{18}$ : rectal temperature $\geqslant 40^{\circ} \mathrm{C}$, disorientation or other significant alterations of mental status, coma, presence of dehydration, or raised serum creatinine phosphokinase (CPK). Clinical features of rhabdomyolysis were verified by limb muscle weakness, pain and tenderness, pigmented urine, and high serum CPK activity. ${ }^{19}$ We measured serum $\mathrm{CPK}$, urea nitrogen, potassium, and calcium within the first 48 hours after admission to hospital and every two to three days thereafter. The peak CPK, urea nitrogen, and potassium values, and lowest calcium values obtained were used for comparison. Electrodiagnostic tests in patients with exertional heat stroke showed a definitive myopathic pattern without neurogenic features.

\section{Muscle biopsy}

Muscle biopsy samples were obtained from the vastus lateralis in 37 patients with exertional heatstroke. The biopsy was taken in the early stage of disease (within 10 days of the heatstroke). Each muscle sample was immediately placed in isopentane which had been well chilled in liquid nitrogen. Serial frozen sections $8 \mu \mathrm{m}$ in thickness were prepared for histopathological and histochemical staining, including haematoxylin and eosin, Gomori trichrome, nicotinamide adenine dinucleotide dehydrogenase (NADH), myophosphorylase, periodic acid Schiff for glycogen, and Sudan black for lipid stains. The muscle fibres were classified as type I, IIA, and IIB on the basis of serial sections stained for myofibrillar ATPase at $\mathrm{pH} 9.4$ after preincubation at $\mathrm{pH} 10 \cdot 3,4 \cdot 6$, and 4.3 . The method was similar to that employed in earlier studies. ${ }^{20}$ Percentage of different fibre types was based on a mean of 637.4 (SD 97.3) classified fibres from each biopsy specimen. The diameter of each fibre type was calculated from 255.9 (SD 17.2) classified fibres. Muscle biopsies from the vastus lateralis were also carried out on the same age group in 15 normal subjects who gave informed consent to serve as controls in this study.

AEROBIC-ANAEROBIC LACTATE THRESHOLD MEASUREMENT

After giving informed consent for the study, 29 patients received a second biopsy with a new skin incision close to that of the first biopsy incision 90-150 (mean 108 (SD 56)) days after exertional heat stroke. These patients also participated in a constant work load test on the treadmill. The treadmill exercise was performed at a speed of $4.0 \mathrm{~m} / \mathrm{s}$. Prolonged exercise tests consisted of a three minute warm up and a 25 minute exercise phase or a test to exhaustion and were carried out at a $4.0 \mathrm{~m} / \mathrm{s}$ constant speed. The initial warm up phase began at a speed of $2 \cdot 6,3 \cdot 0$, or $3.4 \mathrm{~m} / \mathrm{s}$, depending on the endurance capacity of each subject. Lactate concentrations were assessed at the initial warm up phase and at five minute intervals after the treadmill test. The lactate threshold was determined by examining the blood lactate-power output relation during constant exercise. This cut off point for the lactate threshold was taken as $4 \mathrm{mmol} / \mathrm{l}$ as previously described by Weltman et al. ${ }^{21}$

Values are presented as means (SD). Student's $t$ test was used to compare the means of different groups. Descriptive data were compared by $\chi^{2}$ test. Correlation and linear regressions were performed using a programmed method of least squares. One way analysis of variance (ANOVA) was used to analyse differences between groups for a given variable. $P<0.05$ was considered significant.

\section{Results}

The study population consisted of 52 recruits. They were divided into three groups for comparison; group A: exertional heatstroke with rhabdomyolysis $(n=24)$; group B: exertional heatstroke without rhabdomyolysis $(n=13)$; and group $C$ : normal control group $(n=15)$. The criteria for rhabdomyolysis were based on clinical features in addition to muscle biopsy. Twenty four recruits showing positive histological changes of rhabdomyolysis also had typical clinical features of rhabdomyolysis; $12 / 24(50 \%)$ had rhabdomyolysis in the first 24 hours; and all 24 (100\%) developed rhabdomyolysis within three days. The range of peak rectal temperatures was 40.0 to 41.5 (mean $40.4(\mathrm{SD} 0.4))^{\circ} \mathrm{C}$ in 37 patients with exertional heat stroke. Table 1 shows these data together with peak CPK, urea nitrogen, and potassium concentrations, and lowest calcium values in the three groups. There was a significant difference at CPK, blood urea nitrogen, potassium, and calcium concentrations among the three groups. A significant difference also existed between mean values

Table 1 Clinical and biochemical data for three groups of patients and normal subjects

\begin{tabular}{|c|c|c|c|c|c|c|}
\hline & $A(n=24)$ & $B(n=13)$ & $C(n=15)$ & $P(A N O V A)$ & \multicolumn{2}{|c|}{$P($ students $t)$} \\
\hline $\begin{array}{l}\text { Age }(\mathrm{y}) \\
\mathrm{Body} \text { temperature } \\
\mathrm{Ca}^{2+}(\mathrm{mg} / \mathrm{dl})\end{array}$ & $\begin{array}{r}20.4(1 \cdot 1) \\
40.5(0 \cdot 4) \\
7.0(0 \cdot 7)\end{array}$ & $\begin{array}{r}20 \cdot 2(1 \cdot 0) \\
40 \cdot 1(0 \cdot 1) \\
8 \cdot 8(0 \cdot 4)\end{array}$ & $\begin{array}{r}20 \cdot 7(1 \cdot 5) \\
36 \cdot 6(0 \cdot 3) \\
9 \cdot 1(0 \cdot 2)\end{array}$ & $\begin{array}{l}\text { NS } \\
<0.01 \\
<0.01\end{array}$ & $\begin{array}{l}\text { NS } \\
<0.01 \\
<0.001 \\
<0.05\end{array}$ & $\begin{array}{l}\mathrm{A} v \mathrm{~B}, \mathrm{~B} v \mathrm{C} \\
\mathrm{A} v \mathrm{~B}, \mathrm{~A} v \mathrm{C} \\
\mathrm{B} v \mathrm{C}\end{array}$ \\
\hline $\begin{array}{l}\mathrm{K}^{+}(\mathrm{mEq} / \mathrm{l}) \\
\mathrm{BUN}(\mathrm{mg} / \mathrm{dl})\end{array}$ & $\begin{array}{c}4 \cdot 6(0 \cdot 4) \\
75 \cdot 1(22 \cdot 1)\end{array}$ & $\begin{array}{r}4 \cdot 1(0 \cdot 2) \\
28 \cdot 8(8 \cdot 5)\end{array}$ & $\begin{array}{r}4 \cdot 1(0 \cdot 2) \\
11 \cdot 9(2 \cdot 5)\end{array}$ & $\begin{array}{l}<0.01 \\
<0.01\end{array}$ & $\begin{array}{l}<0.001 \\
<0.001\end{array}$ & $\begin{array}{l}\mathrm{A} v \mathbf{B}, \mathbf{A} v \mathbf{C} \\
\mathrm{A} v \mathbf{B}, \mathbf{A} v \mathbf{C} \\
\mathrm{B} v \mathrm{C}\end{array}$ \\
\hline CPK (U/1) & $16710(21632)$ & $1209(1117)$ & $186(43)$ & $<0.01$ & $\begin{array}{l}<0.005 \\
<0.01\end{array}$ & $\begin{array}{l}\mathrm{A} v \mathrm{~B}, \mathrm{~A} v \mathrm{C} \\
\mathrm{B} v \mathrm{C}\end{array}$ \\
\hline
\end{tabular}

Values are means $(\mathrm{SD}) ; \mathrm{BUN}=$ blood urea nitrogen; group $\mathrm{A}$, patients with exertional heatstroke and rhabdomyolysis; group $\mathrm{B}$ patients with exertional heatstroke without rhabdomyolysis; group $\mathrm{C}$, normal subjects. 
Figure 1 Muscle biopsy from a patient with exertional heat stroke in the acute stage showing (A) necrotic fibres which are partially devoid of interfibrial structure in some necrotic fibres and hypercontrature fibres; (B) myophagocytosis. Gomor trichrome stain $\times 200$.

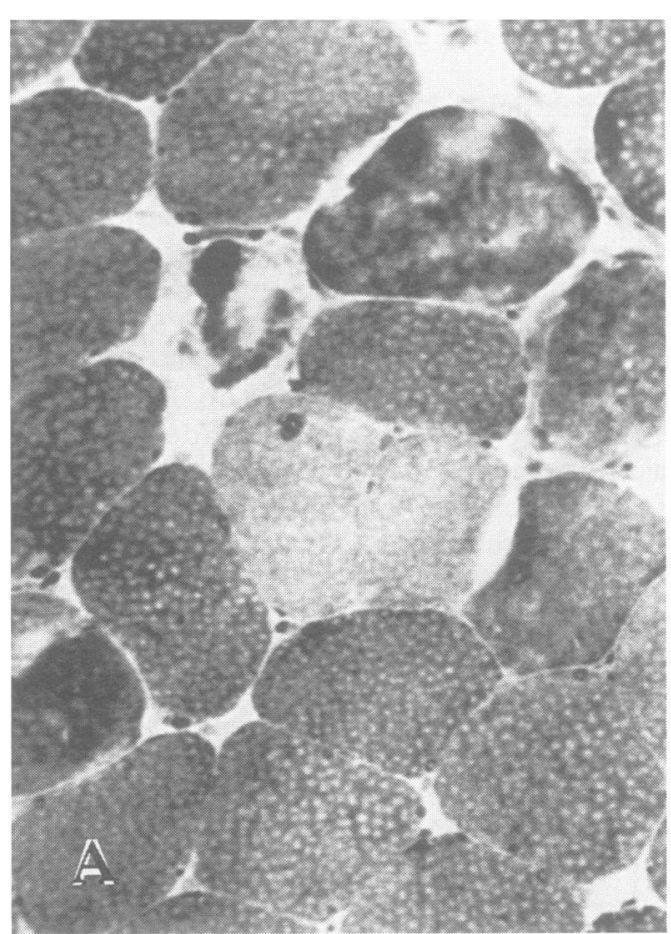

for each laboratory test (table 1). Peak serum CPK was significantly higher in group $A$ than in groups $B$ and $C(P<0.005)$. Blood urea nitrogen concentration was significantly higher in group $A$ than in groups $B$ and $C$, and in group $B$ it was higher than in group $C$ $(P<0.001)$. The mean serum calcium was significantly lower in group A than in groups B and $C(P<0.001)$, and that in group $B$ was lower than that in group $C(P<0.05)$. Serum potassium was also significantly higher in group $A$ than in groups $B$ and $C(P<0.001)$.

The correlation between peak serum CPK and calcium concentrations in 37 patients with exertional heatstroke demonstrated that as peak serum CPK increased, there was a corresponding decrease in serum calcium $(t=$ $-4.79, r=-0.63, P<0.01)$. A positive correlation between peak serum CPK and potassium concentrations was found in 37 patients with exertional heatstroke $(t=3 \cdot 80$, $r=0.54, \mathrm{P}<0.01)$.

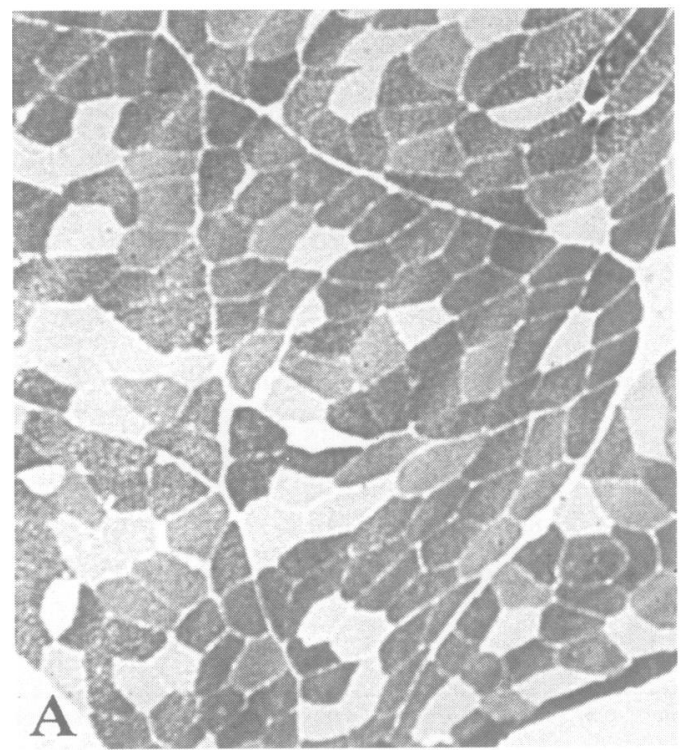

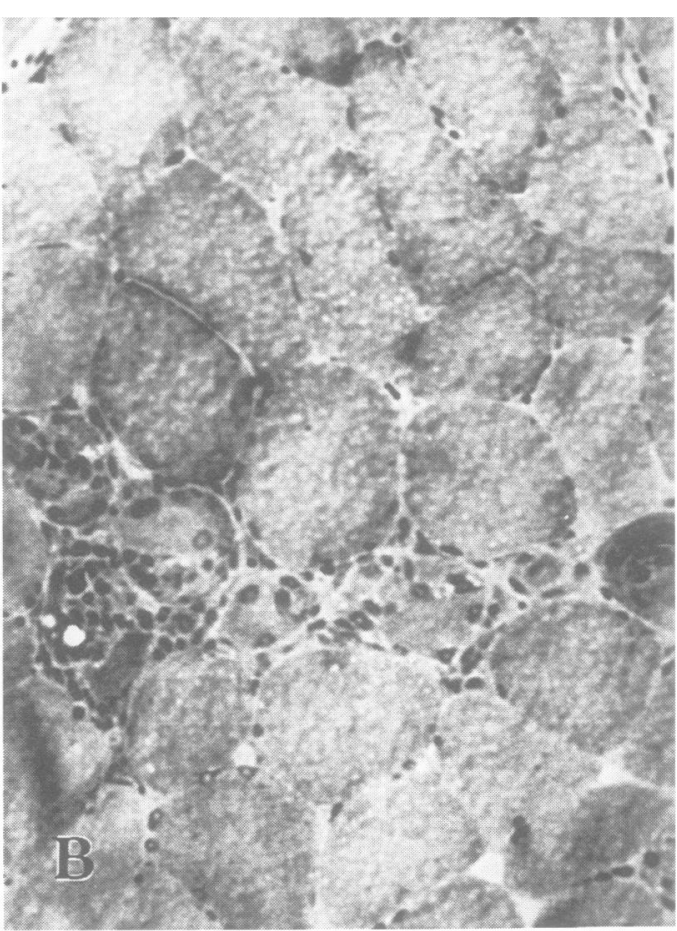

Muscle biopsy from 37 patients with exertional heatstroke and 15 normal subjects demonstrated that 24 patients with heatstroke had rhabdomyolysis showing necrotic, hypercontracture fibres, and occasionally focal inflammatory reactions (fig 1). A major finding was type II fibre predominance (fig 2; the criteria were those reported previously ${ }^{22}$ ) in 22 patients with exertional heatstroke, 18 of whom also had rhabdomyolysis. The percentage of type II fibres was much higher in the exertional heatstroke group than in normal young recruits $(P<0.001$; table 2$)$. Two patients with a history of possible metabolic myopathy developed rhabdomyolysis, but not type II fibre predominance. One of the two patients was found to have ragged-red fibres. He probably had mitochondrial myopathy because of his experience of exercise intolerance with cramping, which had occurred since the age of 9 , and he showed limb weakness, lactic acidaemia $(5.4 \mathrm{mmol} / \mathrm{l})$, and myopathic

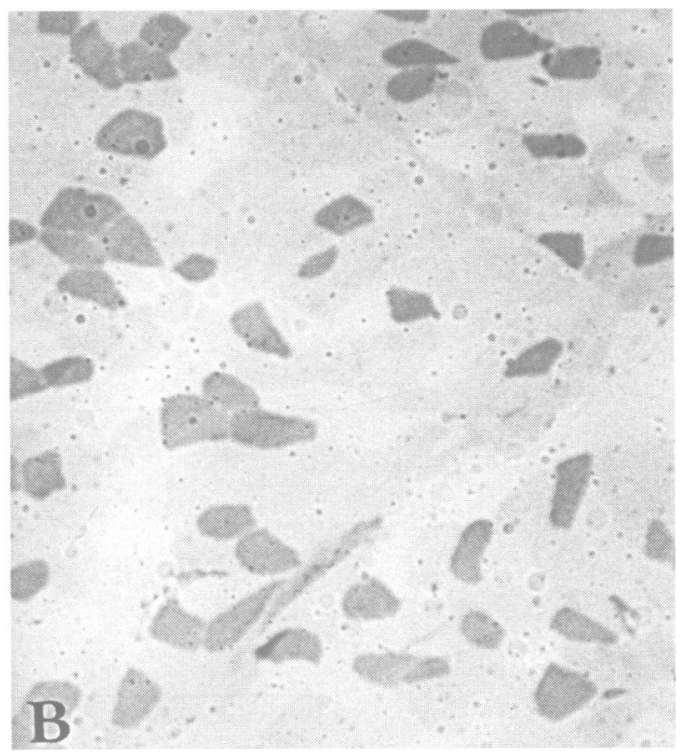

Figure 2 Muscle biopsy of an exertional heat stroke patient showed type II fibre predominance $(91 \%)$. (A) ATPase pH 10.3, (B) ATPase $\mathrm{pH}$ 4.3. Originally $\times 80$ 
Table 2 Mean diameter and percentage of various muscle fibre types in two groups of patients with exertional heatstroke and normal control subjects

\begin{tabular}{|c|c|c|c|c|}
\hline & $A(n=24)$ & $B(n=13)$ & $C(n=15)$ & $P(A N O V A)$ \\
\hline \multicolumn{5}{|l|}{ Fibre types (\%): } \\
\hline Type I & $19 \cdot 0(0 \cdot 1)$ & $24 \cdot 5(5 \cdot 3)$ & $33 \cdot 8(4 \cdot 2)$ & $<0.01$ \\
\hline Type IIA & $26.9(4.9)$ & $29 \cdot 2(5 \cdot 0)$ & $35 \cdot 5(4 \cdot 0)$ & $<0.01$ \\
\hline Type IIB & $54 \cdot 1(7 \cdot 7)$ & $46 \cdot 2(9 \cdot 1)$ & $30 \cdot 7(3 \cdot 8)$ & $<0.01$ \\
\hline Type II (IIA + IIB) & $81 \cdot 1(5 \cdot 1)$ & $75 \cdot 5(5 \cdot 3)$ & $66 \cdot 2(4 \cdot 2)$ & $<0.01$ \\
\hline \multicolumn{5}{|c|}{ Diameter of fibre types $(\mu \mathrm{m})$ : } \\
\hline Type I & $59 \cdot 0(2 \cdot 1)$ & $57.9(1.8)$ & $59 \cdot 1(1 \cdot 8)$ & NS \\
\hline Type IIA & $58 \cdot 7(2 \cdot 7)$ & $57 \cdot 8(2 \cdot 0)$ & $59.8(2 \cdot 0)$ & NS \\
\hline Type IIB & $55 \cdot 8(1 \cdot 7)$ & $53.8(1.6)$ & $56 \cdot 2(2 \cdot 7)$ & NS \\
\hline Type II (IIA + IIB) & $55.9(1.7)$ & $54 \cdot 1(1 \cdot 5)$ & $56.0(1 \cdot 5)$ & NS \\
\hline
\end{tabular}

Values are means $(\mathrm{SD})$

For description of groups A-C, see table 1 .

Table 3 A comparison* of percentage of type II fibres between patients with exertional heatstroke with and without rhabdomyolysis $(n=37)$

\begin{tabular}{lll}
\hline & With rhabdomyolysis & Without rhabdomyolysis \\
\hline Type II fibres $\geqslant 80 \%$ & 18 & 4 \\
Type II fibres $<80 \%$ & 6 & 9 \\
\hline${ }^{\star} \chi^{2}=6.84, P<0.01$. & &
\end{tabular}

EMG findings during a stay in hospital, although he denied having any history of stroke, seizure, or myoclonic seizures. Brain MRI and EEG were negative. The other patient showed an increase in glycogen content in fibres without being myophosphorylase deficient in the muscle biopsy. He also had experienced exercise intolerance since the age of 12. An ischaemic tolerance test was negative. Results from this study suggest that type II fibre predominance found in exertional heatstroke may only occur in non-active people but not in those with myopathy.

Table 3 shows a comparison of rhabdomyolysis in muscle biopsies obtained from patients with exertional heatstroke with and without type II fibre predominance. Patients with exertional heat stroke with type II fibre predominance had a tendency to develop rhabdomyolysis $\left(\chi^{2}=6.84, \quad P<0.01\right)$. In addition, a positive correlation was found between peak serum CPK concentration and percentage of fibre type II in 37 patients with exertional heatstroke (fig $3 ; t=-2 \cdot 9, r=$ $0.44, P<0.01$ ). Peak serum CPK concentration and percentage of fibre type $I$ in 37 patients with exertional heatstroke showed a negative correlation $(t=3.80, r=0.54$, $\mathrm{P}<0.01)$. The diameter of type I and type II fibres was not significantly different in the three groups of subjects $(P<0 \cdot 1$, table 2$)$.

Figure 3 Correlation between serum CPK concentration and percentage of type II fibres in 37 patients with exertional heatstroke . Dashed lines are $95 \%$ confidence intervals.
A second muscle biopsy in 29 patients with exertional heatstroke (excluding the two patients possibly having mitochondrial and glycogen disorders) did not show the noninflammatory necrosis and hypercontracture fibres found in the first biopsy. The percentage of fibre types was not significantly different from the previous biopsy (type $\mathrm{I}, t=1 \cdot 69$, $\mathrm{P}>0.1$; type II, $t=0.48, \mathrm{P}>0.5)$. However, predominance of type II fibres was still found in the 17 subjects who showed this previously. None of these findings occurred in normal healthy subjects (fig 4). Routine mitochondrial enzyme (succinate dehydrogenase, cytochrome C oxidase) glycogen (periodic acid Schiff), and lipid (oil red) reactive stains in muscle biopsies had ruled out the possibility of metabolic muscular disorders.

All subjects (29 previous exertional heat stroke and 15 control subjects) studied completed the entire course of the treadmill work load without problems or side effects. We found a positive correlation between the highest value of blood lactate and the percentage of fibre type II in subjects tested (fig 5). The time required to reach the blood lactate threshold $(4 \mathrm{mmol} / \mathrm{l})$ after a constant treadmill work load was significantly shorter in patients with exertional heat stroke with type II fibre predominance (fig 6). The mean values of blood lactate at each point of exercise time was significantly different in the three groups of subjects after the third minute $(F=28.05,17 \cdot 78$, $27 \cdot 29,36 \cdot 46$, and $29 \cdot 61$ at the 8 th, 13 th, 18 th, $23 \mathrm{rd}$, and 28th minute respectively, $\mathrm{P}<0.01$ ). Moreover, the mean lactate concentration in exertional heat stroke with type II fibre predominance was significantly higher than that in patients without type II fibre predominance or the control subjects $(P<0.01)$.

\section{Discussion}

The normal percentage of type I to type II fibres in the vastus lateralis is about $1: 2$. If the type II fibres are further subdivided, the type I, type IIA, and type IIB fibres each comprise about one third. ${ }^{22}$ In our normal young adults, the percentage of three fibre types was also about one third in each fibre type (mean, 34: 35: $31 \%$ ). But an increased percentage of type II fibres was found in our patients with exertional heatstroke, and nearly $60 \%(22 / 37)$ of patients had type II fibre predominance in their muscle biopsy. We do not know whether type II fibre predominance in our recruits is pathological or hereditary. It is known that type I fibre predominance tends to be associated with the myopathic conditions and particularly with the genetically determined dystrophies, and type II fibre predominance may be associated with motor neuron disease. ${ }^{22}$ In our patients, the clinical features and EMG did not show any evidence of motor neuron disorder.

Previous studies have shown that pathogeneses of exertional heatstroke might originate in two factors; one is the haemodynamic displacement of blood to the periphery during 
Figure 4 Muscle biopsy of the vastus lateralis showing an increased percentage of fibre type II in patients with exertional heat stroke $(A)$ compared with normal subjects $(B)$. ATPase pH 10.3, ( $A$ and $B)$. Originally $\times 80$.

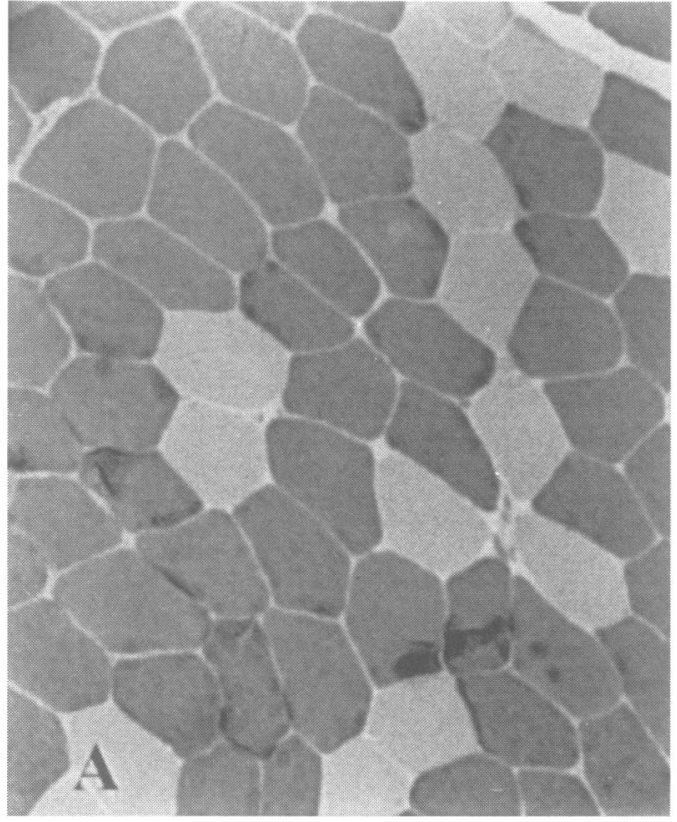

heat stress; the second is mechanical muscle injury with secondary excessively metabolic heat generation. ${ }^{2324}$ Based on this assumption, skeletal muscle with a poor work efficiency (ratio between external work, mechanical work, to the total metabolic rate) might be an important factor in heat intolerance. ${ }^{11}$ It is important to know which type of muscle disorder tends to have poor work efficiency. In this study, we found only two patients with metabolic myopathies (one mitochondria disorder, one glycolytic disorder) in the group with exertional heatstroke. Therefore we could not draw any conclusions, but other studies have also reported that exercise intolerance is one of the clinical features in metabolic myopathies. Therefore, we think that recruits with metabolic myopathy are susceptible to exertional heatstroke during military training. In addition, we found that recruits with type II fibre predominance tended to have a poor endurance capacity under a treadmill load test that might have contributed to the susceptibility to exertional heat stroke and rhabdomyolysis. Further evidence could support this speculation; firstly, the recruits with a higher percentage of type II fibres had a higher serum CPK concentration during the exertional heatstroke episode; secondly, the recruits with type II fibre predominance had a greater tendency towards rhabdomyolysis during the exertional heatstroke episode; thirdly, the recruits with

Figure 5 Correlation between blood lactate concentration and percentage of type II fibres in 29 patients with exertional heat stroke and 15 control subjects. Dashed lines are $95 \%$ confidence intervals.

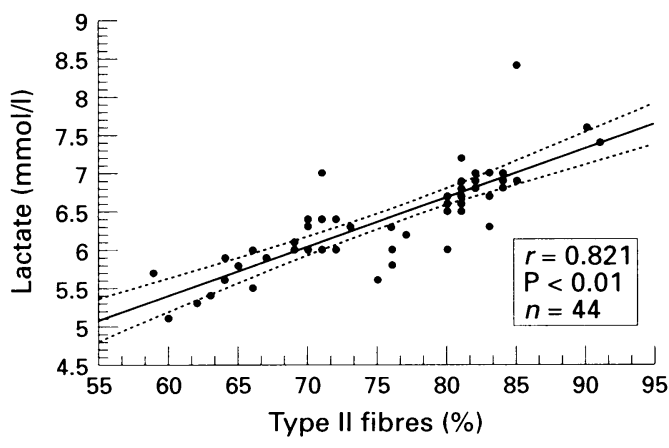

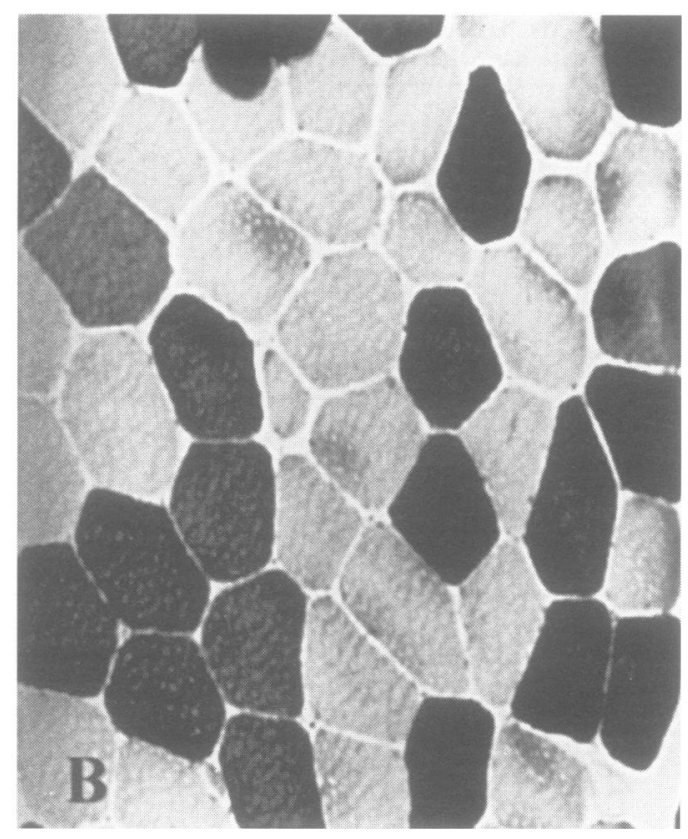

type II fibre predominance rapidly reached the blood lactate threshold during continuous exercise.

Information from other studies about fibre characteristics in different physical performance concentrations could explain our results. Glenmark et $a l^{16}$ reported that the more type I fibres and less type II fibres in adult woman, the stronger the woman. BallBurnett et al ${ }^{14}$ noted that subjects with more type I fibres and less type II fibres also had a longer exercise time to exhaustion during endurance exercise. The question we asked is what different metabolic responses would occur in humans with different percentages of type I and II muscle fibres during exercise. At the level of the muscle cell, ${ }^{14}$ lactate concentration is higher in type II fibres compared with type I fibres during prolonged submaximal exercise (endurance exercise), but glycogen degradation is most pronounced in type $I$ fibres. We know that the lactate product comes from the anaerobic process of glycolysis, and more lactate production means more anaerobic processes, which results in low work efficiency due to less ATP production than during the aerobic process. In continuous treadmill exercise, we also found that the blood lactate concentrations in patients with higher percentages of type II fibres were higher than those in patients with a low percentage These results suggest that the more type II fibres in recruits would result in a lower endurance capacity during exercise.

Blood lactate threshold was first described by Mader et al $^{25}$ in 1976 as the transition area between the purely aerobic to partially anaerobic lactate coverage of the metabolic energy needs of the muscles under given loading conditions. In this study, we found that the time required to reach blood lactate threshold in patients with exertional heatstroke with type II fibre predominance was significantly shorter than that in patients with exertional heatstroke but without type II fibre predominance, and normal healthy subjects. Therefore, patients 


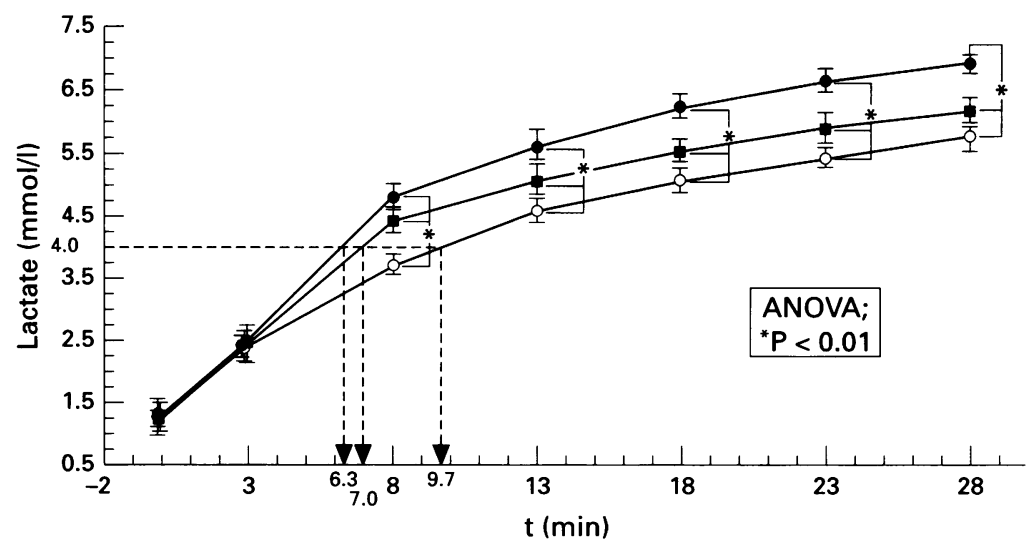

Figure 6 Comparison of blood lactate changes during a constant treadmill test in three different groups of subjects: $\bigcirc$ mean of lactate change in 15 normal subjects; mean of lactate change in 17 patients with exertional heatstroke with type II fibre predominance; - mean of lactate change in 12 patients with exertional heatstroke without type II fibre predominance; dashed line indicates the time required to reach the lactate threshold ( $4 \mathrm{mmol} / \mathrm{l}$ ) in three groups.
3 Larsson L-E, Linderholm $\mathrm{H}$, Muller $\mathrm{R}$, Ringqvist $\mathrm{T}$ Sornas $R$. Hereditary metabolic myopathy with paroxysmal myoglobinuria due to abnormal glycolysis. $\mathcal{f}$ Neurol Neurosurg Psychiatry 1964;27:361-80.

4 Salmon SE, Turner CE. McArdle's disease presenting as convulsions and rhabdomyolysis. Am $\mathcal{F}$ Med 1965;39: 142-8.

5 Patten BM, Wood JM, Harati Y, Hefferan P, Howell RR Familial recurrent rhabdomyolysis due to carnitine palmityltransferase deficiency. $A m$ f $M e d$ 1979;67: 167-78.

6 Engel WK, Vick NA, Glueck CJ, Levy RI. A skeletal muscle disorder associated with intermittent symptoms and a possible defect in lipid metabolism. $N$ Engl $f \mathrm{Med}$ 1970;82:697-794.

7 Gamboa ET, Eastwood AB, Hayes AP, Maxwell J, Penn AS. Isolation of influenza virus from muscle in myoglobinuric polymyositis. Neurology 1979;29:1323-5.

8 Henrich WL, Prophet D, Knochel JP. Rhabdomyolysis associated with Escherichia coli septicemia. South Med $\mathcal{F}$ associated with

9 Ho KJ, Scully KT. Acute rhabdomyolysis and renal failure in Weil's disease. $f$ Med Sci 1980;17:133-7.

10 Kiessling WR, Ricker K, Pflughaupt KW, Mertens HG Haubitz I. Serum myoglobin in primary and secondary skeletal muscle disorders. $\mathcal{F}$ Neurol $1981 ; 224: 229-33$.

11 Epstein Y. Heat intolerance: predisposing factor or residua injury? Med Sci Sports Exerc 1990;22:29-35.

12 Strydom NB. Heat intolerance; its detection and elimination in the mining industry. $S$ Afr $\mathcal{F} S c i$ 1980;76:154-6.

13 Mero A, Jaakkola L, Komi PV. Relationships between muscle fiber characteristics and physical performance capacity in trained athletic boys. F Sports Sci 1991;9:161-71.

with predominantly type II fibres might have a lower endurance capacity than others. Army training is medium to long endurance work; recruits with type II fibre predominance may not tolerate hard work and this would lead to a tendency to develop rhabdomyolysis or exertional heatstroke.

In summary, the occurrence of rhabdomyolysis is a serious complication of exertional heatstroke. Several biochemical markers had been implicated as predictors for the development of rhabdomyolysis. Our muscle biopsy data showed that in type II fibre predominance or underlying myopathy the subject was susceptible to exertional heatstroke and rhabdomyolysis. Blood lactate threshold seems to be a good indicator of the endurance capacity of recruits before training or in patients under recovery. The procedure of measuring blood lactate threshold is a convenient and safe method for predicting the risk of rhabdomyolysis in exercise intolerance. This study was supported partially by the National Science National Defense Medical Research, Republic of China.

1 Rowland LP, Dunne PB, Penn AS, Maher E. Myoglobin and muscular dystrophy. Arch Neurol 1968;18:141-50.

2 Rowland LP, Blake DM, Hirano M, et al. Clinical syndromes associated with ragged red fibers. Rev Neurol 1991;147:467-73.
14 Ball-Burnett M, Green HJ, Houston ME. Energy metabolism in human slow and fast twitch fibers during prolism in human slow and fast twitch fibers durin
longed cycle exercise. $\mathcal{F}$ Physiol 1991;437:257-67.

15 Greenhaff PL, Nevill ME, Soderlund K, et al. The metabolic responses of human type I and II muscle fibers during maximal treadmill sprinting. $\mathcal{f}$ Physiol 1994;478 149-55.

16 Glenmark B, Hedberg G, Kaijser L, Jansson E. Muscle strength from adolescence to adulthood-relationship to muscle fiber types. Eur 7 Appl Physiol 1994;68:9-19.

17 Heck H, Mader G, Mucke S, Muller R, Hollmann W. Justification of the 4-mmol/L threshold. Int $\mathcal{F}$ Sports Med 1985;6:117-30.

18 Shieh SD, Lin YF, Lu KC, Li BL, Chu P, Shyh TP, Diang LK. Role of creatine phosphokinase in predicting acute renal failure in hypocalcemic exertional heat stroke. $A m \mathcal{J}$ Nephrol 1992;12:252-8.

19 Layzer RB. Diagnosis of neuromuscular disorders. In: Layzer RB, ed. Neuromuscular manifestations of systemic Layzer RB, ed. Neuromuscular manifestations

20 Schantz P, Billeter R, Henriksson J, Jansson E. Training induced increase in myofibrillar ATPase intermediate fibers in human skeletal muscle. Muscle Nerve 1982 5:628-36

21 Weltman A, Snead D, Stein P, Seip R, Shurrer R, Rutt R, Weltman J. Reliability and validity of a continuous increthreshold, fixed blood lactate concentrations, and $\mathrm{VO}_{2}$

22 Dubowitz V. Definition of pathological changes seen in muscle biopsies. In: Dubowitz V, ed. Muscle biopsy: practical approach. 2nd ed. Bailliere Tindall Press, practical approach. 2 :

23 Hubbard RW. An introduction: the role of exercise in the etiology of exertional heatstroke. Med Sci Sports Exerc 1990;22:2-5.

24 Rowell LB. General principles of vascular control. In: Rowell LB, ed. Human circulation: regulation during physical stress. New York: Oxford University Press, 1986:363-406. Hollmann W. Zur Beurteilung der sportartspezifischen Ausdauerleistungsfahigkeit. Sportarzt Sportmed 1976, 27(4,5):80-8:109-12. mental treadmill protocol for the determination of lactate max. Int $\mathcal{F}$ Sports Med 1990;11:26-32.

25 Mader A, Liesen H, Heck H, Philippi H, Schurch PM, 